\title{
Symmetry and shape of reconstructed two-dimensional islands
}

\author{
Konstantin Romanyuk, Vasily Cherepanov, and Bert Voigtländer ${ }^{*}$ \\ Peter Grünberg Institut (PGI-3) and JARA-Fundamentals of Future Information Technology, Forschungszentrum Jülich, \\ D-52425 Jülich, Germany
}

(Received 22 December 2010; revised manuscript received 24 March 2011; published 18 May 2011)

\begin{abstract}
We show that the symmetry of reconstructed two-dimensional islands on reconstructed surfaces can deviate from both the symmetry of the underlying substrate and the symmetry of the reconstruction. Only an analysis of the symmetry of the combined system of the reconstruction on the substrate and the reconstruction on the island can predict the symmetry of the island shapes (equilibrium shape or steady-state growth shape). We introduce a general method for the symmetry analysis of the combined system which identifies all possible mutual shifts of the reconstruction on island which obey a certain crystal symmetry.
\end{abstract}

DOI: 10.1103/PhysRevB.83.205413

PACS number(s): 68.35.B-, 68.55.A-, 61.50.Ah

\section{INTRODUCTION}

For equilibrium crystal shapes there is a strict relationship between the symmetry of the equilibrium shape of a crystal and the point-group symmetry of the atoms in the crystal structure. The symmetry of the equilibrium shape of a crystal obeys the point-group symmetry of the crystal structure. ${ }^{1}$ Equilibrium shapes of three-dimensional (3D) crystals $^{2,3}$ were studied using scanning electron microscopy, while equilibrium shapes of two-dimensional (2D) islands were studied by scanning tunneling microscopy (STM). ${ }^{4,5}$ Also for the shapes occurring during the growth of a crystal ("growth shapes of crystals") it is often found that the symmetry of the shape is related to the crystal structure. Usually the symmetry of islands at surfaces is intuitively related to the symmetry of the substrate. For instance, islands on a threefold symmetric $\left(C_{3 v}\right)$ substrate have threefold symmetry. This intuitive relation is often true, but there are exceptions as noticed in our recent work. ${ }^{6}$ In this study a threefold symmetric $\mathrm{Si}(111)$ substrate was used to grow $2 \mathrm{D} \mathrm{Si}$ or Ge islands whose crystal structure is also threefold symmetric. Both substrate surface and island surface were terminated by a threefold symmetric $(\sqrt{3} \times \sqrt{3}) R 30^{\circ}$ reconstruction of $\mathrm{Bi}$. In spite of all these threefold symmetries occurring, the symmetry of the shape of the resulting $2 \mathrm{D}$ islands was observed to have a rhomb shape (for the case of Ge islands) and an even more strange arrow shape in the case of 2D Si islands. Due to the reconstruction present on both substrate and the mutually shifted reconstruction on the island, the threefold symmetry of the system is broken to a $C_{s}$ symmetry.

In this paper we will present a general analysis of the symmetry of reconstructed 2D islands on reconstructed surfaces. We start with the analysis of the inner symmetry of the crystal lattice of the system. The crystal lattice of the system consists of that of the semi-infinite bulk lattice, plus the lattice of the reconstructed surface layer, plus the lattice of the reconstructed island layer, both extended infinitely parallel to the surface. As a result of this we predict the symmetry of the shape of the islands. It will be shown that the shape of the reconstructed islands cannot be predicted from the symmetry of the reconstructed substrate or the symmetry of the reconstructed island alone. The analysis of the symmetry of the combined system of reconstructed substrate and reconstructed island is required in order to predict the symmetry of the shapes of the 2D islands, either growth shapes or equilibrium shapes. We identify for which mutual shifts between the reconstructions on island and substrate a certain symmetry of the combined system is obeyed. For these mutual shifts also the shape of the $2 \mathrm{D}$ islands obeys the same symmetry.

\section{RELATION BETWEEN INNER SYMMETRY OF THE CRYSTAL AND THE SYMMETRY OF THE SHAPE OF THE CRYSTAL}

In the equilibrium case the inner symmetry of a crystal (the symmetry of the crystal lattice) and the symmetry of the equilibrium shape of the crystal are the same $\left(S_{\text {lattice }}=S_{\text {shape }}\right){ }^{1}$ Stated a bit differently, the point-group symmetry of the crystal lattice is the same as the point-group symmetry of the crystal shape. Therefore, if we know the symmetry of the crystal structure (inner symmetry of the crystal), we are able to predict the symmetry of the shape of the crystal.

For the case of crystal growth the relation between inner symmetry and symmetry of the shape is not as clear. We will see in the following that the symmetry of the shape of the crystal can be higher or lower or the same as the inner symmetry of the lattice. Therefore, it seems hopeless that one can derive conclusions on the symmetry of the growth shape from an analysis of the inner symmetry. However, in the following we will specify the conditions under which inner symmetry of a crystal and the symmetry of the shape of the crystal are identical as in the equilibrium case. Under these conditions straight conclusions about the symmetry of growth shapes of crystals can be obtained from an analysis of the inner symmetry of the crystal.

The case in which the growth shape of the crystal is lower than the crystal symmetry occurs if crystal growth proceeds under external conditions which have lower symmetry than the crystal lattice. For instance, if the deposition of new material occurs preferably from one side, this side of the crystal will grow faster and the crystal shape will be influenced by this. This is actually a special case of the Curie principle, ${ }^{7}$ which states that a system with symmetry $B$ under the influence of an external perturbation of symmetry $E$ has symmetry $B \cap E$. Another case in which a lower symmetry of the growth shape occurs is if initially the island nucleus has an unsymmetrical shape. The transient growth period which is influenced by the shape of the nuclei turns into the steady 
state if the majority of the islands have adopted the shape of larger islands. Here we consider the "steady-state growth shape" after possible transients related to the nucleation have died out. In the following we will exclude such asymmetric external conditions or initial conditions. Under this condition the crystal shape can have the same or higher symmetry as the underlying crystal structure.

Generally the relation between the symmetry of a crystal (without low-symmetry external conditions) and its physical properties is stated in Neumann's principle. ${ }^{8}$ If a crystal is invariant under certain symmetry operations, any of its physical properties must also be invariant with respect to the same symmetry operations. This means that the physical properties obey the same (or higher) symmetry as the inner symmetry of the crystal. The Neumann principle can be also stated in terms of point-group symmetries: The symmetry of the crystal is a subgroup of the symmetry of a certain physical property ( $\left.S_{\text {crystal }} \subseteq S_{\text {physprop }}\right)$. To see that the symmetry of a physical property can be higher than the crystal symmetry, consider, for instance, a scalar physical property such as the temperature (or density). The temperature has infinite symmetry (invariant under all point-group symmetry operations). In our case the growth shape of the crystal is the physical property under study.

A wrong conclusion about the symmetry of the shape of the crystal could be obtained if the term "symmetry of the shape of a crystal" is not defined properly. The "macroscopic definition" would be the following: If a symmetry operation (for instance, mirror operation) is applied to the crystal and the crystal looks "macroscopically" the same after the application of the symmetry operation, then we can say that the crystal obeys this symmetry "macroscopically." On the other hand, the crystal obeys a certain "microscopic" symmetry if also the atomic structure (i.e., structure of the crystal faces) is the same before and after the symmetry transformation.

A simple example for the difference between macroscopic and microscopic symmetry is given by the minicrystal shown in Fig. 1. Looking at the shape of this crystal from afar (not seeing the atoms), it has a cubic shape, while when looking at it at the atomic level, the symmetry is lower than the cubic symmetry. In order to obtain correct and consistent conclusions we will consider the microscopic symmetry of the shape in the following.

For the case of growth shapes we will assume that the growth speed of the crystal in a certain direction depends

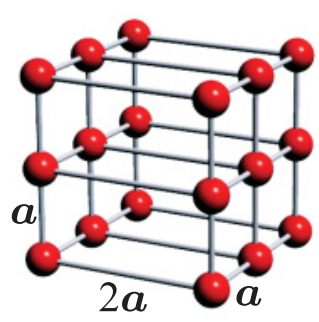

FIG. 1. (Color online) This figure shows the difference between macroscopic symmetry and microscopic symmetry. Looking at the shape of this crystal from afar, it has a cubic shape, while when looking at it on the atomic level, the symmetry is lower than the cubic symmetry. only on the local surface orientation (step orientation in 2D). This assumption was introduced by Frank ${ }^{9}$ and means that the growth speed is given by the function $v(\theta)$ in $2 \mathrm{D}$, which can be called the kinematic Wulff plot, ${ }^{10}$ in close analogy to the equilibrium Wulff plot. ${ }^{11}$ Due to the one-toone correspondence between the equilibrium Wulff plot and the kinetic Wulff plot, the inner symmetry of the crystal is the same as the symmetry of the growth shape. If one would consider the macroscopic shape, the predicted symmetry of islands would be the same or higher than the one which we predict here.

The formation of facets is not yet explained by this, and a threefold internal symmetry could result in any threefold symmetric crystal shape, for instance, also a cloverleaf shape. A simplified explanation for the formation of facets is as follows. If there are minima (cusps) in the function $v(\theta)$, this leads to the formation of facets (straight step edges in 2D), ${ }^{10}$ in close analogy to the formation of facets in the equilibrium shape of crystals due to cusps in the surface free energy as a function of the direction which is described in the Wulff plot. ${ }^{12}$

Going back to our case of an island on a substrate and making the above-mentioned assumptions that (a) the outer conditions are symmetric (i.e., have at least the inner symmetry of the crystal), (b) we consider the steady-state growth shape without initial transients related to the nucleation, (c) the growth speeds depend only on the surface orientation by the function $v(\theta)$, and (d) when we consider the microscopic structure, the following general statement can be made: The point-group symmetry of the growth shape of an island is identical to the point-group symmetry of the underlying crystal structure. Therefore, in the following, we will analyze the inner symmetry of the crystal structure of an island (plus substrate) in order to predict the symmetry of the (growth) shape of the islands.

\section{SYMMETRY OF RECONSTRUCTED 2D ISLANDS}

A surface reconstruction can be formed by the rearrangement of the atoms of the top layer(s) and/or by different atoms (adsorbed or deposited) on top of the surface. In the horizontal direction the surface reconstruction unit cell can have a size which is larger than the projection of the bulk unit cell onto the surface. In the vertical direction the surface reconstruction unit cell includes all layers which are different from the bulk layers. The difference in the bulk can be a different chemical nature of the atoms, a difference in the atom positions, or a difference in the bond angles. Regarding the relation of the reconstruction to the substrate, we consider only commensurate reconstructions. This means that any (atom) position within the reconstruction unit cell has a well-defined relation to the substrate. In physics terms the reconstruction atoms are bonded to the substrate, resulting in a fixed relationship between both (e.g., on top or bridge site). The symmetry of the reconstructed substrate $(R S)$ is given by the intersection $\operatorname{Symm}(R S)=\operatorname{Symm}(R) \cap \operatorname{Symm}(S)$, with $R$ and $S$ being the reconstruction and the substrate, respectively.

Now we will consider the symmetry of the combined system $(C)$ of reconstructed substrate $(R S)$ and reconstructed 2D island $(R I)$. The island is considered as pseudomorphic, i.e., the lattice structure of the substrate is continued in the one 


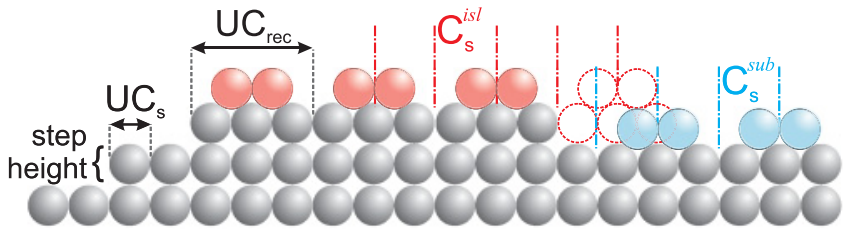

FIG. 2. (Color online) Cross section through the combined system of reconstructed substrate and reconstructed island (three times reconstruction on a simple cubic lattice). The substrate reconstruction has a $C_{s}$ mirror symmetry as well as an island reconstruction. However, due to a shift between both reconstructions (for the translational domain shown here) these mirror planes are not identical (shifted) and the combined system obeys no mirror symmetry. We say that the mirror symmetry of the combined system is broken.

atomic layer higher island. The symmetry of the combined system is given by the intersection of the symmetry elements of both subsystems $\operatorname{Symm}(C)=\operatorname{Symm}(R S) \cap \operatorname{Symm}(R I)$. A symmetry element which is contained in the intersection is a symmetry element of the first and the second system. In building this intersection we have to consider that the symmetry element is defined by both its type (e.g., mirror symmetry or twofold rotation symmetry) and its position (e.g., position of the mirror plane or position of the rotation axis).

In the following we analyze the inner symmetry of the combined system of a reconstructed substrate and a reconstructed pseudomorphic 2D island, which has an atomic step height relative to the substrate as shown in Fig. 2.

Now we apply this general statement to the $1 \mathrm{D}$ example in which a $(3 \times 1)$ reconstruction is present on the substrate, as well as on the island (Fig. 2). At first sight one might think that the symmetry of the combined system is simply that of the $(3 \times 1)$ reconstruction. However, this is not generally the case as shown in Fig. 2. The set of symmetry elements on the substrate reconstruction $[\operatorname{Symm}(R S)]$ can be different from the symmetry elements of the same reconstruction on the island $[\operatorname{Symm}(R I)]$ due to a shift between both. We have to remember that symmetry elements are only identical if they are of the same type (e.g., mirror plane) and if their position is identical. The positions of the two corresponding mirror planes $C_{s}^{\text {sub }}$ and $C_{s}^{\text {isl }}$ are shifted as indicated in Fig. 2. Therefore, none of the mirror planes in Fig. 2 is a mirror plane of the combined system.

This effect, where the number of symmetry elements for the combined system is lower than the number of symmetry elements for each subsystem, we call symmetry breaking or lowering of the symmetry of the combined system. In the nomenclature used above, $\operatorname{Symm}(R S) \neq \operatorname{Symm}(R I) . R S$ and $R I$ have the same number of the same types of symmetry elements, however, the positions of the symmetry elements are shifted with respect to each other. For the intersection, both types and positions of the symmetry elements have to be considered. An equivalent to the intersection of two sets of symmetry elements is the set of symmetry elements which are common for the two subsystems (common symmetry elements).

In a practical analysis of the inner symmetry of the combined system, we consider infinite lattices of the island reconstruction and the reconstruction of the substrate layer, as indicated by the dashed lines in Fig. 2 .

In the following we discuss several case studies of the analysis of the symmetry of the combined system of reconstructed substrate and island, and deduce predictions for the island shapes. In particular, we will use the fact that, starting from the symmetry analysis of the individual subsystems, the symmetry of the combined system is obtained by the finding which symmetry elements are common symmetry elements for all subsystems. This is a simple way to analyze if the symmetry of the combined system (reconstructed island plus reconstructed substrate) is lower than the symmetry of each of the two subsystems ("symmetry breaking").

\section{EXAMPLES OF THE SYMMETRY ANALYSIS FOR SPECIFIC SURFACES}

\section{A. Symmetry analysis for reconstructions on the simple cubic (100) surface}

The first case we discuss is the (100) surface of the simple cubic lattice. The unit cell is indicated by a large red square and the mirror planes (black lines) are indicated in Fig. 3(a), while the fourfold rotation axes are shown as small red squares.

For the (100) surface of the simple cubic lattice, the lattice of a one (or also $N$ ) atomic layer high island is not shifted relative to the substrate. This makes the analysis simpler but is not generally true for other cases. In the following we analyze the symmetry of the combined system of reconstructed substrate layer and island.

In the simplest case of a $(1 \times 1)$ reconstruction on the substrate layer and island, the $C_{4 v}$ symmetry of both subsystems is also saved for the combined system. On the other hand, for any reconstruction breaking the $C_{4 v}$ symmetry of the substrate, the symmetry of the combined system is broken (lowered) right from the beginning. We do not consider these obvious cases


FIG. 3. (Color online) (a) Top view on the (100) plane of the simple cubic lattice. The unit cell is indicated in red and the atoms are indicated as red balls. Also the symmetry elements are indicated. (b) $(2 \times 2)$ reconstructed substrate layer (blue) and $(2 \times 2)$ reconstructed island (red) obeying both a $C_{4 v}$ symmetry but mutually shifted horizontally by one $(1 \times 1)$ surface lattice constant [the $(1 \times 1)$ unit cell is indicated by dashed lines in the upper left-hand side]. The symmetry of the combined system is broken to $C_{2 v}$. No common diagonal mirror planes exist (dashed lines). 
here, but only reconstructions which preserve the point-group symmetry of the substrate. When there is no mutual shift present between the reconstructions on the island layer and substrate layer, obviously, the symmetry of the combined system is preserved. The fact that a $C_{4 v}$ symmetry present on the substrate layer reconstruction and island reconstruction is not generally preserved for the combined system can be seen in the example shown in Fig. 3(b). Here the $(2 \times 2)$ reconstruction which obeys the $C_{4 v}$ symmetry of the substrate (indicated by arrows) is shifted horizontally by one (substrate) lattice unit on the island (red) relative to the reconstruction of the substrate layer (blue). Analyzing the symmetry of the combined system, one can see that the diagonal mirror planes (dashed line) are no more mirror planes for the combined system and the symmetry of the combined system is broken to $C_{2 v}$. This means that the symmetry of the shape of the islands will be lowered to a $C_{2 v}$ symmetry. Therefore, in general, on a simple cubic (100) surface a $C_{4 v}$ symmetric $(2 \times 2)$ surface reconstruction can break the $C_{4 v}$ of the substrate. The symmetry will be lowered to a $C_{2 v}$ symmetry if the reconstructions are shifted in one direction (horizontally or vertically) by one substrate lattice unit. For the case where there is no phase shift between the island and substrate $(2 \times 2)$ reconstruction (or the same shifts in both directions), the $C_{4 v}$ symmetry will be preserved for the combined system and therefore also for the island shape. In total, for half of the four possible shifts of the reconstructions the symmetry is broken to a $C_{2 v}$ symmetry while for the other half the $C_{4 v}$ symmetry is retained. This analysis can be extended to the general case of a $(2 N \times 2 N)$ reconstruction. Only for no shift or a simultaneous shift in both horizontal and vertical directions by $N$ substrate lattice units is the $C_{4 v}$ symmetry retained. For all other cases of shifts by integer values of the substrate lattice constant the symmetry is broken.

\section{B. Symmetry analysis for reconstructions on the fcc(100) surface}

The next case which we will consider is the fcc (100) surface. which is shown in Fig. 4(a) as a ball model and obeys $C_{4 v}$ symmetry. For this crystal surface the upper (island) layer is shifted inherently relative to the one atomic layer lower (substrate) layer by $a / \sqrt{2}$ ( $a$ is the surface lattice constant) along the diagonal of the surface unit cell, as indicated in Fig. 4(a).

While we performed the symmetry analysis in the previous case explicitly by analysis of particular mirror planes, we present now another method which is often easier to apply in particular for more complicated cases. We are searching for symmetry elements which are common symmetry elements for both subsystem island and substrate layers. Common symmetry elements are symmetry elements which have the same type (e.g., $C_{4}$ ) and the same position (e.g., rotation axis or mirror plane for the case of mirror symmetry). In order to find the point-group symmetry of the combined system, one has to find all common symmetry elements and identify the point-group symmetry of the common symmetry elements from this.

In Fig. 4(b), a $(2 \times 2)$ surface reconstruction unit cell which obeys the $C_{4 v}$ symmetry of the substrate is indicated schematically by arrows. In order to facilitate the search for

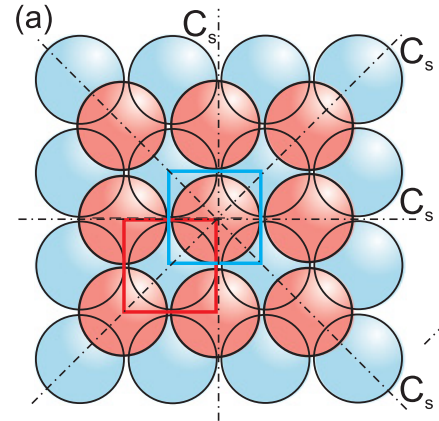

(b)

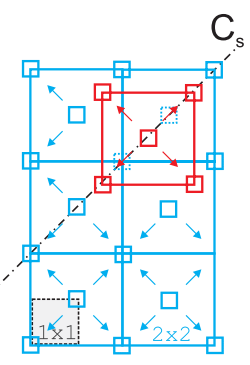

FIG. 4. (Color online) Top view on a ball model of the fcc (100) plane. The surface unit cells of the substrate and island are indicated by blue and red squares, respectively. Adjacent layers are inherently shifted by $a / \sqrt{2}$ along the diagonals of the surface unit cell. (b) Symmetry diagram for the $C_{4}$ symmetry element for the case of a $(2 \times 2)$ reconstruction. The $C_{4}$ symmetry elements are marked as small squares on the substrate layer (blue) and the island (red). The $(1 \times 1)$ unit cell is marked by dashed lines in the lower left-hand corner. Due to the intrinsic lateral shift between the subsequent atomic layers, no common $C_{4}$ symmetry element exists and the $C_{4 v}$ symmetry is broken for the combined reconstructed system, to a $C_{s}$ symmetry, with a mirror plane shown as a dashed line.

common symmetry elements of both subsystem island and substrate layers, we use symmetry diagrams such as the one shown in Fig. 4(b) for the $C_{4}$ symmetry element. Here the positions (rotation axes) for the $C_{4}$ symmetry elements on the substrate layer reconstruction (blue) and island reconstruction (red) are indicated by small squares. The larger squares show the $(2 \times 2)$ unit-cell boundaries. Due to the inherent noninteger mutual shift between the surface lattices of two adjacent (100) layers, also the island reconstruction is shifted by $a / \sqrt{2}$ along the diagonal of the surface unit cell relative to the substrate layer reconstruction. As a result of this there exist no common $C_{4}$ symmetry elements of substrate layer reconstruction and island reconstruction, as can be seen in Fig. 4(b), and the $C_{4}$ symmetry is always broken. However, as can be also seen from the symmetry diagram, there exist common mirror planes along a direction indicated in Fig. 4(b) by a dashed line. The mirror planes which were present in the other three directions on island reconstruction or the substrate layer reconstruction are no longer mirror planes of the combined system. There exist two possible domains for this mutual shift of the reconstructions between the island layer and substrate layer with a $C_{s}$ mirror plane along one of the two diagonal mirror planes. It turns out that for any $(N \times N)(N>1)$ reconstruction on the fcc (100) surface, the symmetry of the combined system is reduced to $C_{s}$ and correspondingly the shape of the $2 \mathrm{D}$ islands will have $C_{s}$ symmetry.

The symmetry analysis presented in this section is incomplete and has several drawbacks. Each translational domain has to be considered separately. In order to avoid tedious studies for all possible cases, we will present in the following a general method of the analysis of common symmetry elements. We will evaluate all symmetry elements and determine if a certain symmetry is preserved by the combined system of a reconstructed substrate plus a reconstructed island. Therefore, the general analysis does not only predict if a symmetry is 

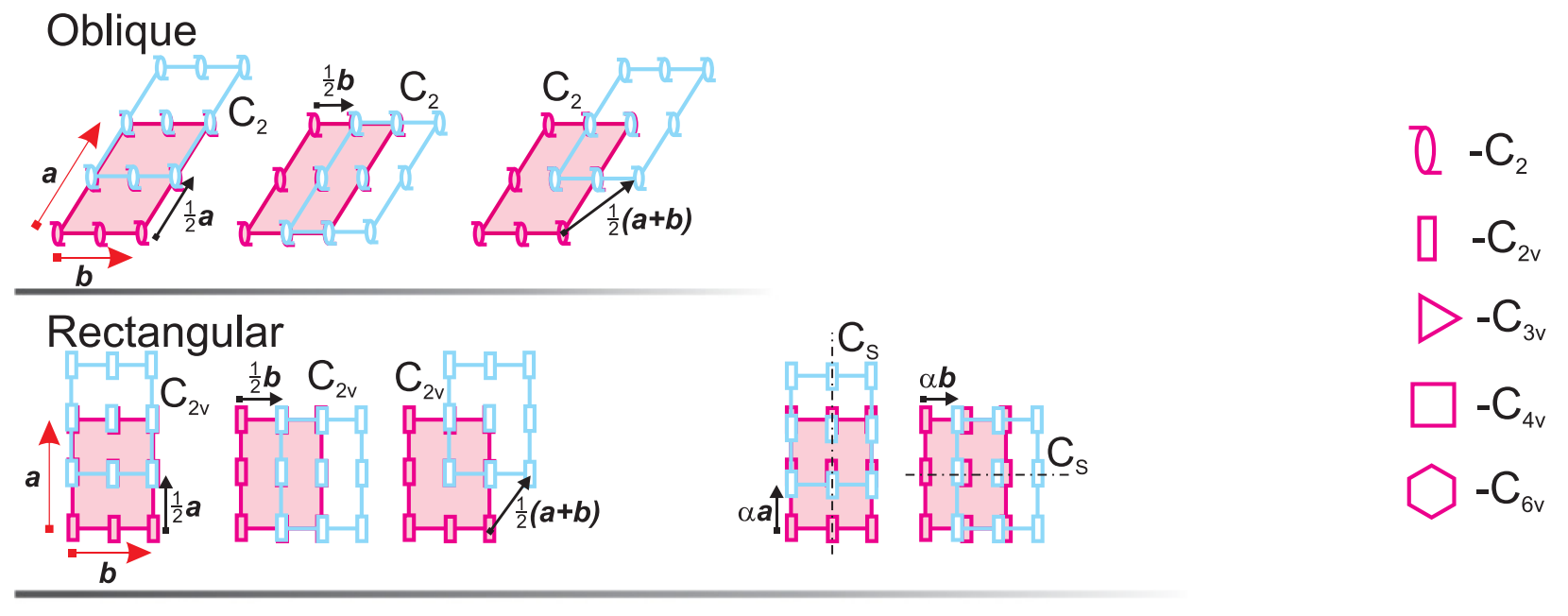

\section{Rectangular centered}
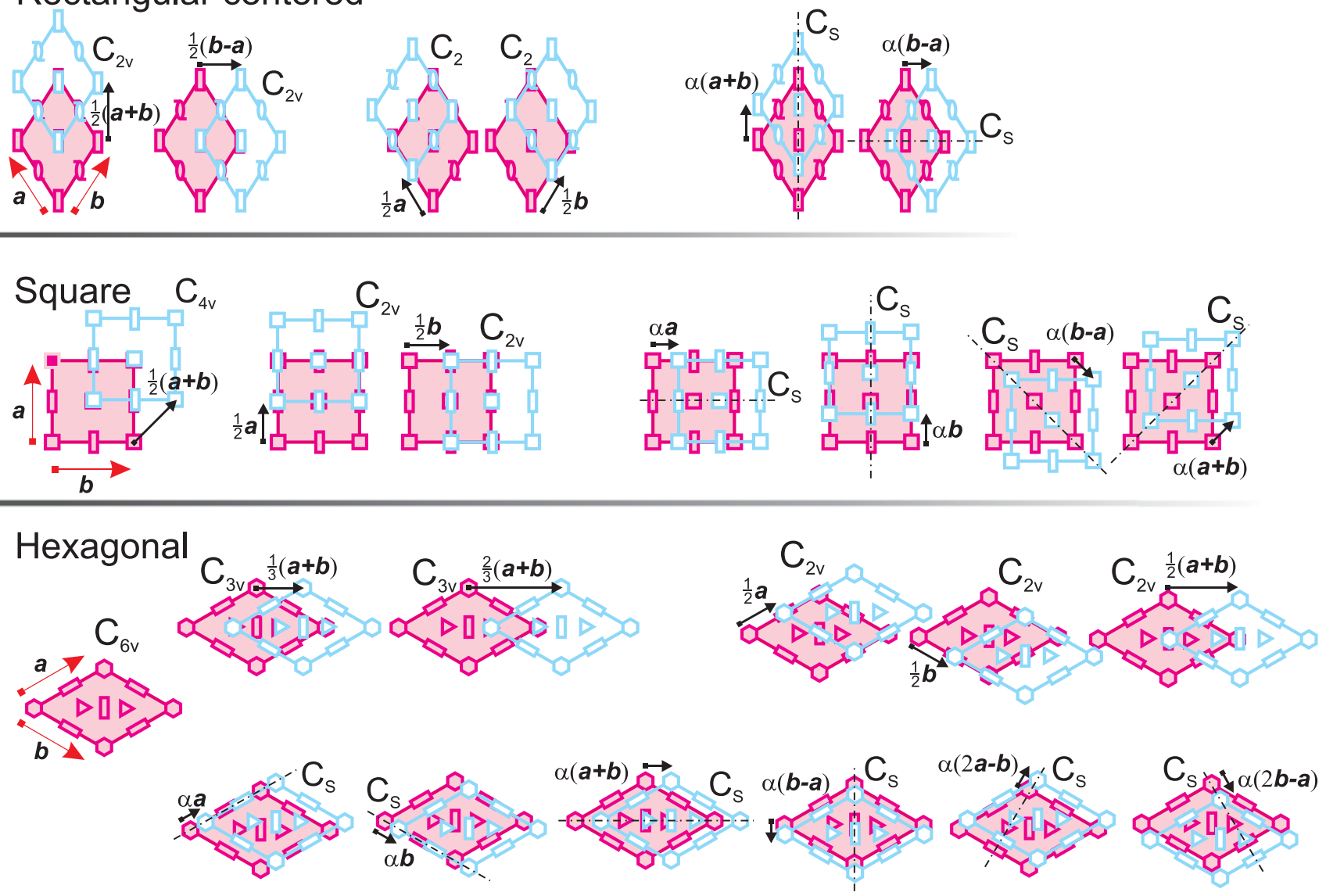

FIG. 5. (Color online) Possible relative lateral shifts of two Bravais lattices which preserve a certain symmetry for the combined system. This symmetry is indicated for each shift by the respective symbol (e.g., $C_{2 v}$ ). In the left-hand part of the rows, the shifts which preserve the symmetry of the individual lattices are shown. Toward the right-hand side of each row, shifts with lower symmetry are shown. The symmetry elements are marked by symbols of their symmetry. The two lattices can be identified with the substrate layer reconstruction and the island reconstruction, respectively.

preserved or broken, but is also able to predict to which lower symmetry the symmetry is broken in specific cases.

\section{GENERAL METHOD OF SYMMETRY ANALYSIS}

Here we present an outline of a general method of symmetry analysis before we apply it to examples. The task is to identify all possible cases of combined systems with a reconstruction on the substrate layer $(R S)$ and the same reconstruction, mutually shifted, on the island layer $(R I)$, which obey a certain inner crystal symmetry of the combined system. The symmetry of a combined system is naturally given by the intersection of the symmetry elements of both subsystems $C=R I \cap R S$ (or stated differently by the common symmetry elements). A 
TABLE I. Shifts between two reconstruction unit cells which preserve the indicated symmetry for the combined system. Zero shift means any shift which is a multiple of the basis vectors. The basis vectors $a$ and $b$ are indicated in Fig. 5. For any other shifts the symmetry is lost, i.e., only identity $(I)$ is the symmetry element of the combined system. This table defines the shift vectors $\vec{V}_{\text {rec }}$ in Eq. (1).

\begin{tabular}{lcc}
\hline \hline Lattice & Symmetry & Mutual lateral shifts \\
\hline Oblique & $C_{2}$ & $0, \frac{1}{2} \vec{a}, \frac{1}{2} \vec{b}, \frac{1}{2}(\vec{a}+\vec{b})$ \\
Rectangle & $C_{2 v}$ & $0, \frac{1}{2} \vec{a}, \frac{1}{2} \vec{b}, \frac{1}{2}(\vec{a}+\vec{b})$ \\
Centered rectangle & $C_{s}$ & $\alpha \vec{a}, \alpha$ \\
& $C_{2 v}$ & $0, \frac{1}{2}(\vec{a}+\vec{b}), \frac{1}{2}(\vec{b}-\vec{a})$ \\
& $C_{2}$ & $0, \frac{1}{2} \vec{a}, \frac{1}{2} \vec{b}$ \\
Square & $C_{s}$ & $\alpha(\vec{a}+\vec{b}), \alpha(\vec{b}-\vec{a})$ \\
& $C_{4 v}$ & $0, \frac{1}{2}(\vec{a}+\vec{b})$ \\
Hexagon & $C_{2 v}$ & $0, \frac{1}{2} \vec{a}$ \\
& $C_{s}$ & $\alpha \vec{a}, \alpha \vec{b}, \alpha(\vec{a}+\vec{b}), \alpha(\vec{b}-\vec{a})$ \\
& $C_{6 v}$ & $0, \frac{1}{3}(\vec{a}+\vec{b}), \frac{2}{3}(\vec{a}+\vec{b})$ \\
& $C_{3 v}$ & $0, \frac{1}{2} \vec{a}, \frac{1}{2} \vec{b}, \frac{1}{2}(\vec{a}+\vec{b})$ \\
& $C_{2 v}$ & $\alpha \vec{a}, \alpha \vec{b}, \alpha(\vec{a}+\vec{b}), \alpha(\vec{b}-\vec{a}), \alpha(2 \vec{a}-\vec{b})$, \\
\hline \hline
\end{tabular}

brute force way is to analyze the symmetry of all possible combined systems (all translational domains) individually. In the following we describe a method which we think is a "more simple" and general way to accomplish the task given above in order to predict the symmetry of island shapes. This general method consists of three steps.

In the first step of our general method we consider the symmetry properties of the reconstruction Bravais lattice (without basis). Generally the surface reconstruction lattice has to be one of the five $2 \mathrm{D}$ Bravais lattices, ${ }^{13}$ which are mentioned in Fig. 5, together with their symmetry elements marked by the respective symbols. For clarity we left out the mirror planes explicitly. Implicitly they are included in the $C_{2 v}, C_{3 v}, C_{4 v}$, and $C_{6 v}$ symbols. For instance, the $C_{2 v}$ symbol includes a $C_{2}$ rotation axis at the center of the symbol as well as two mirror planes (horizontal and vertical) through the center of the symbol. In the following we use a shorthand notation in which, for example, the term " $C_{2 v}$ symmetry element" means all symmetry elements included in that group, i.e., the $C_{2}$ rotation, the horizontal and the vertical $C_{S}$ mirror planes, and the identity operation.

Now we consider two Bravais lattices of the same type shifted relative to each other. In the second step of our symmetry analysis we identify the shifts of the Bravais lattices for which each symmetry element of the shifted lattice lies on top of the same type of symmetry element of the unshifted lattice, as shown in the left-hand part of Fig. 5. This condition for the shifts between the two lattices can also be stated differently: Each symmetry element is a common symmetry element of both (shifted and unshifted) lattices, or the intersection of the symmetry elements contains all symmetry elements of the individual lattices. In these cases the symmetry of the combined system is the same as the symmetry of the individual lattices. By restricting the following analysis only to these few (allowed) shifts we can avoid tedious case studies of all possible mutual translational domains.

For other the shifts toward the right-hand side in Fig. 5 the symmetry of the combined system does not preserve the symmetry of the original lattice. However, here one can analyze to which symmetry the symmetry of the combined system is reduced (broken). For some shifts a symmetry element of the red lattice falls on top of a symmetry element of the blue lattice. If the symmetry elements which lie on top of each other are of the same type, these common symmetry elements are then symmetry elements of the combined system. If the symmetry elements which lie on top of each other are of a different type, the symmetry of the combined system at this point is reduced to the intersection of the respective symmetries of the two (blue and red) subsystems. For instance, for the square Bravais lattice, the vertical shift of the blue lattice by half a surface lattice constant results in a situation in which a $C_{2 v}$ symmetry element (which is shorthand for all symmetry elements of the $C_{2 v}$ symmetry group) of the blue lattice falls on top of a $C_{4 v}$ symmetry element of the red lattice and vice versa. The symmetry of the combined system at these positions is then the intersection of $C_{2 v}$ and $C_{4 v}$ which is $C_{2 v}$. This means that in this case the symmetry of the combined system of both lattices is reduced (broken) to $C_{2 v}$. Or stated differently: The $C_{2 v}$ symmetry element (no longer the $C_{4 v}$ symmetry element) is a common symmetry element of both lattices when they are mutually shifted as described.

The most interesting example arises for the hexagonal Bravais lattice, which has one $C_{6 v}$ symmetry element per unit cell. If the second lattice is shifted horizontally by one third of the horizontal extension of the unit-cell length, a $C_{6 v}$ symmetry element of the unshifted (red) lattice always falls on top of a $C_{3 v}$ symmetry element of the shifted (blue) lattice. The symmetry of the combined system at this point is the intersection between $C_{3 v}$ and $C_{6 v}$, which is $C_{3 v}$. Arbitrary shifts along directions shown as black arrows in Fig. 5 reduce the symmetry of the combined system to the symmetry of a mirror plane $\left(C_{s}\right)$. For all other shifts the symmetry of the combined system is reduced to identity. The results for the mutual shifts between two Bravais lattices shown in Fig. 5 are summarized more quantitatively in Table I.

All mutual shifts between the two Bravais lattices which preserve a certain symmetry can be written as a shift vector $\vec{V}_{\text {rec }}$, which can be expressed as

$$
\vec{V}_{\mathrm{rec}}=\beta \vec{a}+\gamma \vec{b}
$$

where $\vec{a}$ and $\vec{b}$ are the unit-cell vectors of the reconstruction lattice, defined in Fig. 5, and (for a certain symmetry) the coefficients $\beta$ and $\gamma$ have to be taken from Table I as single numbers (or including the parameter $\alpha$ for the mirror planes). Considering a specific lattice and a specific symmetry, the corresponding line in Table I gives all possible shift vectors $\vec{V}_{\text {rec }}$.

For the case when the reconstruction has a basis (inner structure) which has a lower symmetry than the Bravais lattice, the symmetry of the combined system of Bravais lattice and basis has to be considered in steps 1 and 2 , instead of 
the Bravais lattice alone. Here again the common symmetry elements of the combined system of Bravais lattice and basis are given by the intersection of both. In this case certain symmetry elements have to be removed from Fig. 5, which results in less possible mutual shifts, and also some entries in Table I have to be removed in this case.

In Fig. 6 two examples are shown. In Fig. 6(a) a $C_{3 v}$ symmetry of the basis was assumed, which breaks the $C_{6 v}$ point-group symmetry of the Bravais lattice to a $C_{3 v}$ pointgroup symmetry. In Fig. 6(a) the shifts of two lattices which preserve the $C_{3 v}$ symmetry for the combined system of two reconstructed lattices (or break the symmetry to $C_{s}$ ) are shown. Due to this basis there are no $C_{6 v}$ and $C_{2 v}$ symmetry elements, and in Table I for the hexagonal lattice the first $\left(C_{6 v}\right)$ and third $\left(C_{2 v}\right)$ row have to be neglected. Figure 6(b) corresponds to the inclusion of a $(\sqrt{3} \times \sqrt{3})$ basis and will be discussed later.

In the third step we consider if the mutual shifts $\vec{V}_{\text {rec }}$ between the two Bravais lattices identified in step 2 are compatible with the bulk structure of the crystal. On first sight this seems to be a trivial condition which is always fulfilled (as in Fig. 2). However, the stacking sequence of the atomic layers can induce a definite shift between subsequent layers and thus between the surface reconstructions on island and substrate. An example of this is the (111) plane of the diamond structure. For simplicity we would like to discuss the generic situation for an easier 1D model shown in Fig. 7. Here it is easily seen that the (bulk) lattice structure shifts laterally by one half lattice unit when going from one layer to the next. This case we call it a "shift of the bulk lattice" due to a step edge.

If we consider for this case the combined system of the mutually shifted reconstructions identified in step 2, there exist cases in which the corresponding bulk structures (below the island reconstruction and the substrate reconstruction) do not coincide. An example for such a case is shown in Fig. 7(a). The bulk structure is shown in gray and in the left-hand part a one atomic layer high island with a times three reconstruction on top is also shown (also in gray). As can be seen, this reconstruction has two mirror planes per unit cell, indicated by black dashed-dotted lines. The same (laterally not shifted, $\vec{V}_{\text {rec }}=0$ ) reconstruction is shown in blue in Fig. 7(a), moved one layer lower. For this allowed shift between the two reconstruction lattices identified in step 2 , the corresponding bulk lattices do not coincide. Since this is a natural condition which has to be fulfilled by the combined system, we have to exclude this mutual shift from the set of systems which maintain the symmetry of the reconstruction lattice.

For the other case possible from the analysis in step 2 $\left(\vec{V}_{\text {rec }}=1 / 2 \vec{a}\right)$, shown in Fig. 7(b), the two reconstruction lattices are laterally shifted while maintaining the same mirror planes (gray and red). In this case the two bulk lattices below the island and below the substrate coincide. In general, it has to be considered if the mutual shifts of the reconstructions identified in step 2 [Eq. (1)] are consistent with a valid translational shift of the bulk lattice, i.e., leading an identical bulk lattice below the island and substrate. The possible translational vectors of the bulk parallel to the surface can be expressed by the $(1 \times 1)$ surface lattice vectors $\vec{a}_{0}$ and $\vec{b}_{0}$ as $n \vec{a}_{0}+m \vec{b}_{0}$, with $n$ and $m$ being integers. Additionally, however, the lateral shift of the bulk crystal lattice at a step edge $\left(\vec{V}_{\text {step }}\right)$ also has to be considered. The possible lateral shifts between the bulk lattices below the island relative to the one below the substrate (leading an identical bulk lattice below island and substrate) can be expressed as follows:

$$
\vec{V}_{\text {bulk }}=\vec{V}_{\text {step }}+n \vec{a}_{0}+m \vec{b}_{0} .
$$

Only if a shift vector found in Eq. (1) is equal to an allowed bulk shift vector given in Eq. (2), this shift found in step 2 is also a valid bulk shift vector. In order to verify if, for a certain shift vector $\vec{V}_{\text {rec }}$ found in step 2 , a bulk shift vector exists which satisfies the condition

$$
\vec{V}_{\text {bulk }}=\vec{V}_{\text {rec }}
$$

the relation between the $(1 \times 1)$ surface lattice vectors and the reconstruction lattice vectors has to be known. This relation is given by the matrix notation as $\vec{a}=\hat{R} \vec{a}_{0}$ and $\vec{b}=\hat{R} \vec{b}_{0}$, with $\hat{R}$ being the transformation matrix describing the relation between the substrate lattice and the reconstruction lattice. In Ref. 14 a set of unique rules is given to define the unit-cell vectors of the substrate and reconstruction overlayers.

In our 1D example in Fig. 7, the relation between substrate and reconstruction reduces to $\vec{a}=\hat{R} \vec{a}_{0}=3 \vec{a}_{0}$, and the "shift"

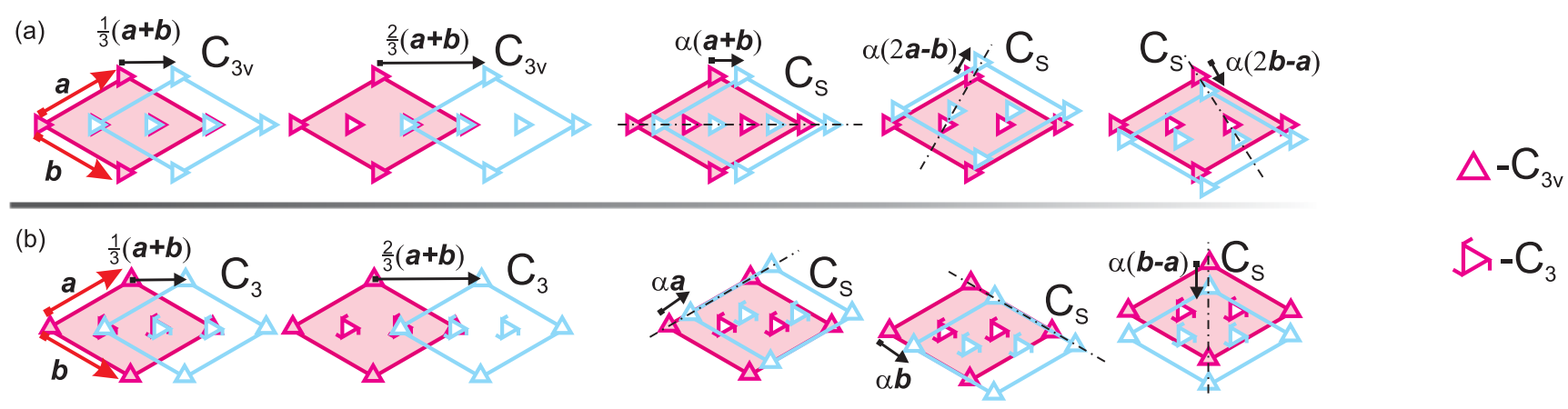

FIG. 6. (Color online) Examples for the inclusion of a basis of lower $\left(C_{3 v}\right)$ symmetry into the symmetry considerations for a hexagonal Bravais lattice. (a) The sixfold rotation symmetry at the corners of the unit cell of the hexagonal Bravais lattice is broken to a $C_{3 v}$ symmetry due to the presence of the $C_{3 v}$ symmetric basis. The shifts of two lattices which preserve the $C_{3 v}$ symmetry for the combined system of two reconstructed lattices (or break the symmetry to a $C_{s}$ symmetry) are shown. (b) The inclusion of a $(\sqrt{3} \times \sqrt{3}) 30^{\circ}$ reconstruction as a basis is shown. This basis has two $C_{3}$ symmetry elements inside the unit cell. 

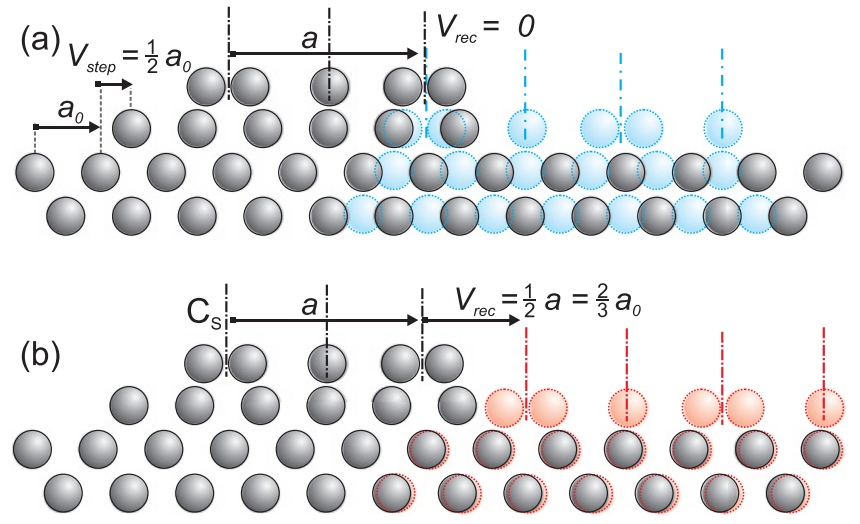

FIG. 7. (Color online) (a) Side view on a surface in which the bulk lattice shifts by one half lattice unit at every step edge. For the reconstruction on the bulk layer (left-hand side) and the (unshifted) reconstruction on the one atomic layer lower substrate layer (righthand side), the mirror planes (gray and blue dashed-dotted lines) are identical. However, this shift (zero) does not lead to the same bulk structure below the island reconstruction (gray) and below the one layer lower substrate reconstruction (blue). Of course, the same bulk structure is an obvious condition for an allowed shift. (b) For a lateral shift of half a reconstruction unit-cell vector the mirror planes (gray and red) coincide as in (a). However, in this case also the bulk structures below the island and substrate layer coincide.

of the bulk crystal parallel to the surface at a step edge is $\vec{V}_{\text {step }}=1 / 2 \vec{a}_{0}$. For the shift $\vec{V}_{\text {rec }}=0$ found in step 2 and shown in Fig. 7(a), the comparison results in

$$
\vec{V}_{\text {rec }}=0 \neq \vec{V}_{\text {bulk }}=\vec{V}_{\text {step }}+n \vec{a}_{0}=1 / 2 \vec{a}_{0}+n \vec{a}_{0} .
$$

There exists no integer value of $n$ for which the condition $\vec{V}_{\text {rec }}=\vec{V}_{\text {bulk }}$ can be satisfied. Therefore, this shift found in step 2 does not satisfy the "bulk condition." For the other shift found in step $2\left[\vec{V}_{\text {rec }}=1 / 2 \vec{a}\right.$, shown in Fig. $7($ b) $]$ the comparison results in

$$
\vec{V}_{\text {rec }}=1 / 2 \vec{a}=3 / 2 \vec{a}_{0}=\vec{V}_{\text {bulk }}=\vec{V}_{\text {step }}+n \vec{a}_{0}=1 / 2 \vec{a}_{0}+1 \vec{a}_{0} \text {. }
$$

Therefore, here the "bulk condition" is satisfied with $n=1$, and this shift, which is identified in step 2, results in "the same bulk" below the island and below the one atomic step lower substrate layer. Generally it has to be checked in step 3 if the possible shift vectors $\left(\vec{V}_{\text {rec }}\right)$ identified in step 2 correspond also to valid bulk translational shift vectors $\left(\vec{V}_{\text {bulk }}\right)$.

As a summary of our general method of the symmetry analysis, we list the three steps below:

(1) Identify the point-group symmetry elements of the reconstruction Bravais lattice (plus reconstruction basis).

(2) Identify possible mutual shifts of the reconstruction lattices $\left(\vec{V}_{\text {rec }}\right)$ for which the combined system has common symmetry elements.

(3) Analyze which shifts identified in step 2 correspond also to valid bulk translational shift vectors, i.e., $\vec{V}_{\text {rec }}=\vec{V}_{\text {bulk }}$.

The cases of possible shifts found in step 2 which do not lead to a valid bulk translational shift vector have to be excluded. Then all possible mutual shifts of the island reconstruction relative to the substrate reconstruction which obey a certain symmetry for the combined system have been found without involving many case studies. If for a certain symmetry no cases (shifts) are left which fulfill the above-mentioned conditions, the combined system does not obey this symmetry and we say this symmetry is broken. For the 1D case treated here explicitly as an example, the general method of symmetry analysis is no simplification, however, in the following examples the general method saves tedious case studies of many translational domains.

\section{A. General symmetry analysis of the diamond structure $(N \times N)$ reconstructed (111) surfaces}

Now we apply our general method of symmetry analysis to the case of a $(N \times N)$ reconstruction on the diamond lattice (111) surface. Here we consider only one of the three possible $120^{\circ}$ rotated domains of the reconstruction. The Bravais lattice of this reconstruction is the hexagonal lattice, and we assume that the basis of the reconstruction obeys the same $C_{3 v}$ symmetry as the diamond structure (i.e., lower symmetry than the Bravais lattice). The positions of the $C_{3 v}$ symmetry elements in the reconstruction unit cell are known from the symmetry to be at the corners of the unit cell and at the centers of the triangular half unit cells, indicated by triangles in the unit cell shown in blue in Fig. 8(a) (step 1 with a lower symmetry basis included). Step 2 of our analysis shows us that there are only three possible independent shifts between the reconstruction unit cells which preserve the $C_{3 v}$ symmetry for the combined system, i.e., lead to a lattice of common $C_{3 v}$ symmetry elements. These three independent shifts of the reconstruction unit cell are indicated in Table I and are shown in Fig. 8(a) as full blue (zero shift, $\left.\vec{V}_{\text {rec }}=0\right)$, dashed red $\left[\vec{V}_{\text {rec }}=1 / 3\left(\vec{a}_{0}+\vec{b}_{0}\right)\right]$, and dotted green lines $\left[\vec{V}_{\text {rec }}=2 / 3\left(\vec{a}_{0}+\vec{b}_{0}\right)\right]$, with the reconstruction unit-cell vectors as defined in Fig. 8(a).

The substrate $(1 \times 1)$ unit cell vectors are introduced in Fig. $8(\mathrm{~b})$ as $\vec{a}_{0}=1 / 2(0 \overline{1} 1)$ and $\vec{b}_{0}=1 / 2(\overline{1} 01)$. The unit vectors of the $(M \times M)$ reconstruction are related to the substrate $(1 \times 1)$ unit cell vectors by the reconstruction matrix $\hat{M}$ as

$$
\left[\begin{array}{l}
a \\
b
\end{array}\right]=\left[\begin{array}{cc}
M & 0 \\
0 & M
\end{array}\right]\left[\begin{array}{l}
a_{0} \\
b_{0}
\end{array}\right],
$$

i.e., $\vec{a}=M \vec{a}_{0}$ and $\vec{b}=M \vec{b}_{0}$.

In the following (step 3 of our analysis), we analyze the lateral shift of the substrate lattice induced by an atomic step on the (111) surface of the diamond structure. A perspective view of the upper two bilayers (red and blue) of the diamond (111) lattice is shown in Fig. 8(c). In each bilayer there exist three inequivalent atomic positions (numbered 1, 2, and 3). As can be seen from the figure, equivalent atomic positions in the two bilayers are shifted laterally by $1 / 3\left(\vec{a}_{0}+\vec{b}_{0}\right)$. Stated a bit differently, the projections of two mutual (111) double layers onto the (111) plane superimpose onto each other if they are shifted by $1 / 3\left(\vec{a}_{0}+\vec{b}_{0}\right)$, or as we stated before more loosely: The "shift of the bulk lattice" at an atomic step edge is $\vec{V}_{\text {step }}=1 / 3\left(\vec{a}_{0}+\vec{b}_{0}\right)$.

In the following we analyze which of the three mutual shifts of the reconstruction lattices are in accord with an allowed bulk 

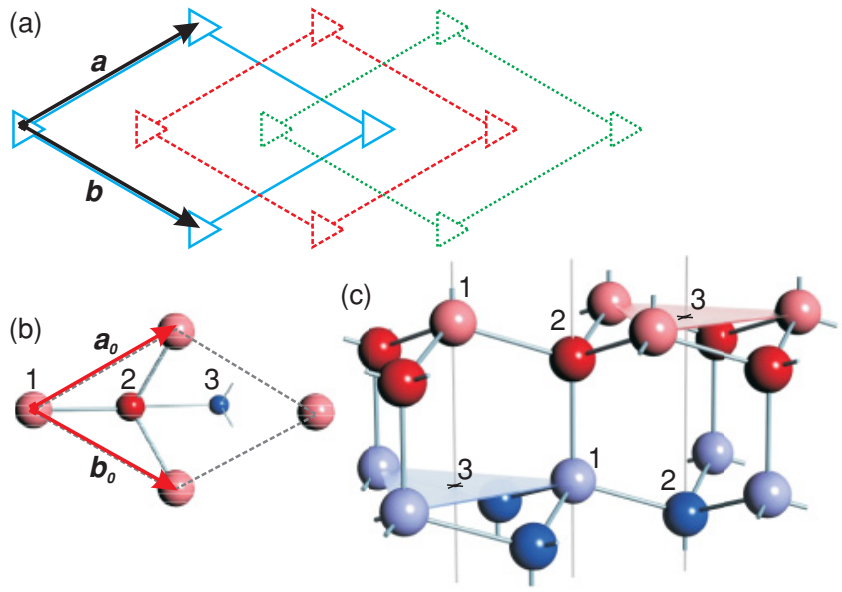

FIG. 8. (Color online) (a) Symmetry analysis of a reconstructed diamond lattice (111) surface. A reconstruction unit cell with $C_{3 v}$ symmetry is shown by blue lines. There are only three independent shifts possible (including zero shift) which result in common $C_{3 v}$ symmetry elements, shown in blue, red, and green. These shifts preserve the original diamond lattice of symmetry elements. The reconstruction unit-cell vectors are indicated. (b) Top view to the unreconstructed diamond lattice (111) surface surface. The substrate surface unit-cell vectors are indicated. The upper bilayer is shown in red, and the lower in blue. (c) Perspective view to the first and second bilayer of the diamond lattice. There exist three inequivalent atomic positions in each bilayer (1,2, and 3). A bilayer high step leads to a lateral shift in the atomic arrangement of $\vec{V}_{\text {step }}=1 / 3\left(\vec{a}_{0}+\vec{b}_{0}\right)$.

translational shift. There exist $N^{2}$ possible shifts (translational domains) between the reconstruction on the island and the reconstruction on the substrate. The possible independent bulk shift vectors are

$$
\vec{V}_{\text {bulk }}=\vec{V}_{\text {step }}+n \vec{a}_{0}+m \vec{b}_{0}=1 / 3\left(\vec{a}_{0}+\vec{b}_{0}\right)+n \vec{a}_{0}+m \vec{b}_{0},
$$

with $n, m=0,1, \ldots, M-1$. Now we have to analyze which of the three previously determined reconstruction shift vectors $\vec{V}_{\text {rec }}$ are consistent with an allowed bulk shift vector, i.e., $\vec{V}_{\text {bulk }}=\vec{V}_{\text {rec }}$. The three previously mentioned reconstruction shift vectors can be written in terms of $\vec{a}_{0}$ and $\vec{b}_{0}$ as

$$
\vec{V}_{\text {rec }}^{1}=0, \quad \vec{V}_{\text {rec }}^{2}=\frac{M}{3}\left(\vec{a}_{0}+\vec{b}_{0}\right), \quad \vec{V}_{\text {rec }}^{3}=\frac{2 M}{3}\left(\vec{a}_{0}+\vec{b}_{0}\right) \text {. }
$$

A valid shift vector also has to be consistent with a bulk shift vector given in Eq. (7). For the case where the reconstruction is a multiple of three (i.e., $M=3 N$ ), the bulk condition Eq. (7) cannot be fulfilled for any of the three $\vec{V}_{\text {rec }}$ given in Eq. (8). Therefore, the $C_{3}$ symmetry is always broken for a $(3 N \times 3 N)$ reconstruction. Only $C_{s}$ mirror symmetries survive for shifts in directions indicated in Fig. 6(a).

For the cases of $(3 N \pm 1 \times 3 N \pm 1)$ reconstructions the $C_{3 v}$ symmetry can be saved for one of the shifts in Eq. (8). A bit loosely explained, one of the shifts of the reconstruction unit cell between substrate and island $\vec{V}_{\text {rec }}^{2}$ or $\vec{V}_{\text {rec }}^{3}$ can be "compensated" by a corresponding shift of the bulk lattice due to an atomic step. In the case of a $(3 N \pm 1 \times 3 N \pm 1)$ reconstruction, the reconstruction on the island can be shifted relative to the reconstruction of the substrate in $(3 N \pm 1)^{2}$ different translational domains. Only for one of these is the $C_{3 v}$ symmetry retained.

\section{B. Application of the symmetry analysis to the diamond structure $(111)(\sqrt{3} \times \sqrt{3})$ surfaces}

Now we analyze the $\sqrt{3}$ reconstructions on the diamond (111) surface which are rotated by $30^{\circ}$ relative to the substrate lattice (Fig. 9). (Also here we consider only one of the three $120^{\circ}$ rotated domains of the reconstruction.) These reconstructions occur, for instance, frequently upon adsorption of metals or semimetals on $\mathrm{Si}(111)$ or Ge (111) surfaces. $^{15}$ In Fig. 9 two examples are shown: $\mathrm{A}(\sqrt{3} \times \sqrt{3}) R 30^{\circ}$ reconstruction unit cell (red shaded area on the left-hand side) and a $(2 \sqrt{3} \times 2 \sqrt{3}) R 30^{\circ}$ reconstruction unit cell (blue shaded area on the right-hand side) are shown together with the underlying diamond lattice. One important point to mention is that for these reconstructions not all rotation axes obey additionally a $C_{3 v}$ symmetry (i.e., additional three mirror planes). The adatoms or sometimes trimers of adatoms are located at the corner of the unit cell. ${ }^{15}$ These rotation axes obey $C_{3 v}$ symmetry, while the other two rotation axes inside the unit cell do obey a $C_{3}$ symmetry (no mirror planes), as can be seen from Fig. 9. The preceding analysis covered step 1 of our general method: symmetry properties of the reconstruction lattice including the basis with $C_{3 v}$ symmetry shown in Fig. 9.

Mutual shifts of these reconstruction lattices are shown in Fig. 6(b). Only for the two mutual shifts shown in the left-hand part of Fig. 6(b) (plus the zero shift) is a $C_{3}$ symmetry obtained for the combined system (step 2). Here the symmetry of the rotation axes of the combined system is the intersection of $C_{3 v}$ and $C_{3}$, which is $C_{3}$.

In Fig. 9 the unit vectors of the substrate $(1 \times 1)$ surface unit cell and of the reconstruction unit cell are shown. For a $(\sqrt{3} \times$ $\sqrt{3})$ reconstruction the relation between both is $\vec{a}=2 \vec{a}_{0}-\vec{b}_{0}$ and $\vec{b}=\vec{a}_{0}+\vec{b}_{0}$. For a $(N \sqrt{3} \times N \sqrt{3})$ reconstruction a factor $N$ has to be included. Using the substrate unit-cell vectors

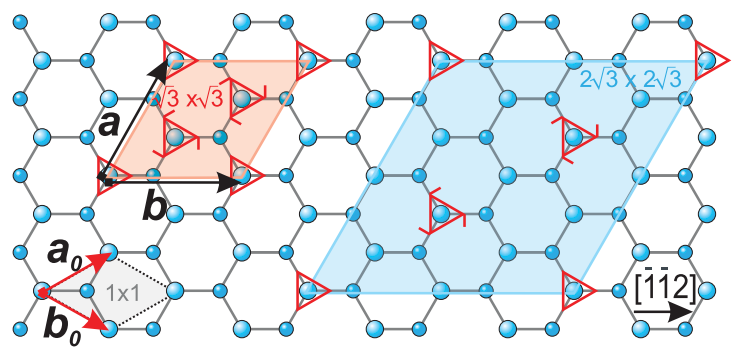

FIG. 9. (Color online) Unit cells of the $(\sqrt{3} \times \sqrt{3}) R 30^{\circ}$ reconstruction (left-hand side) and the $(2 \sqrt{3} \times 2 \sqrt{3}) R 30^{\circ}$ reconstruction (right-hand side) and the underlying diamond lattice (111) surface. The rotation axes at the corners of the reconstruction unit cell are indicated by triangles and obey $C_{3 v}$ symmetry. The rotation axes inside the unit cell have $C_{3}$ symmetry. The unit vectors of the substrate surface and the reconstruction are indicated by arrows. As explained in the text, the symmetry of the combined system of island reconstruction and substrate reconstruction is always broken to a $C_{s}$ symmetry along a [11ㄹ] direction. 
the three possible shifts which maintain the $C_{3}$ symmetry [left-hand part of Fig. 6(b) plus zero shift] can be written for a $(N \sqrt{3} \times N \sqrt{3})$ reconstruction as

$$
\vec{V}_{\text {rec }}^{1}=0, \quad \vec{V}_{\text {rec }}^{2}=N \vec{a}_{0}, \quad \vec{V}_{\text {rec }}^{3}=2 N \vec{a}_{0} .
$$

These relations can be inferred directly from Fig. 9. Comparing these shifts which maintain the $C_{3}$ symmetry to the allowed bulk shifts along the (111) surface of the diamond structure given in Eq. (7), one sees that due to the noninteger factor in Eq. (7) these shift vectors can never have the same value. Due to the noninteger shift induced by the step edge $\left(\vec{V}_{\text {step }}\right)$ the combined system will never have a $C_{3}$ symmetry.

While the $C_{3}$ symmetry is always broken for the combined system, mirror planes can be retained, as will be shown in the following. As can be seen from Fig. 6(b), there are three directions for the mutual shift which preserve the $C_{s}$ symmetry. The corresponding shift vectors for a $(N \sqrt{3} \times$ $N \sqrt{3}$ ) reconstruction can be written in terms of the substrate basis vectors as

$$
\begin{gathered}
\vec{V}_{\text {rec }}^{1}=\alpha^{1} N\left(\vec{a}_{0}+\vec{b}_{0}\right), \\
\vec{V}_{\text {rec }}^{2}=\alpha^{2} N\left(-\vec{a}_{0}+2 \vec{b}_{0}\right), \\
\vec{V}_{\text {rec }}^{3}=\alpha^{3}(\vec{b}-\vec{a})=\alpha^{3} N\left(-2 \vec{a}_{0}+\vec{b}_{0}\right) .
\end{gathered}
$$

The bulk condition for the possible shifts [Eq. (7)] which has to be fulfilled as well limits the number of possible shifts. It turns out that for the $(\sqrt{3} \times \sqrt{3})(N=1)$ reconstruction there are three shifts which fulfill the bulk condition as well. In total, as a result of the symmetry breaking for the $(\sqrt{3} \times \sqrt{3})$ reconstruction, no $C_{3}$ symmetric islands occur, but the island shapes have one mirror plane $\left(C_{s}\right.$ symmetry) along one of the three [11ㄹ] directions. This behavior was indeed observed for the system of $2 \mathrm{D} \mathrm{Si}$ or $\mathrm{Ge}$ islands on $\mathrm{Si}(111)$, where islands and substrates are terminated by a $\mathrm{Bi}(\sqrt{3} \times \sqrt{3}) R 30^{\circ}$ reconstruction, as reported in our previous publication. ${ }^{6}$ In this case islands with only one mirror plane were observed, despite of the fact that a threefold $C_{3 v}$ symmetry was present for the substrate and both the island reconstruction and the substrate reconstruction.

\section{Symmetry and the "real physical world"}

The symmetry analysis performed up to now was a mathematical analysis. The advantage is that such an analysis is always strict and seems to give very definite conclusions. A symmetry is either obeyed or not obeyed (broken). However, the implications of symmetry breaking in the real physical world can range from almost insignificant to a considerable effect. For implications of symmetry breaking in a crystal structure on the physical properties of the crystal an interaction is needed. We would like to discuss this for the example of the growth speed of steps, which we consider here as a physical property. We start with an example of a pseudomorphic 2D island on a surface with a reconstruction initially only present on the surface and not on the island. For this case the symmetry of the combined system is just the reconstruction symmetry. The (pseudomorphic) island cannot induce any symmetry breaking since it is carved from the bulk crystal and obeys its symmetry (compare also Fig. 2). As a consequence of this, also the growth shape of the island will obey the above-mentioned symmetry. If we now introduce a small patch of reconstruction also on the island, the symmetry of the total combined system can be broken immediately, as we discussed in detail before. Such symmetry breaking occurs immediately and strictly already for a small patch of reconstruction present at the center of the island. However, if we think about the implications on the physical properties (step speeds in our case) the consequences of this symmetry breaking can be infinitesimal. If the reconstruction patch on the island is far from the island step edges, the interaction between this patch and the step edges is minute and the step speeds are hardly influenced by the reconstruction patch on the island. Consequently (strict) symmetry breaking has virtually no influence on the shape of the crystal. Therefore, the original symmetry of the growth shape is retained in spite of the fact that the symmetry is now strictly broken. The implication of symmetry breaking on the step speeds becomes more and more significant as the reconstruction patch expands toward the step edges. When the reconstruction is also present at the step edge itself, the influence on the step speed is largest and can lead to island shapes of reduced (broken) symmetry as observed recently. ${ }^{16} \mathrm{~A}$ special case of surface reconstruction present at the step edge is a passivated step edge. ${ }^{17}$ Passivation or depassivation of step edges will have a major influence on the growth speed of the steps, since the step edges are the places were crystal growth is actually happening.

The atomic structure of the zone next to the boundary of the island (the step edges of the island) determines the growth speed of the various step edges of the island. The details of the atomic structure of the step edges are usually not known. However, it is not necessary to know the details of the atomic structure in order obtain conclusions related to symmetry. Since the atomic structure of step edges in directions related by the point-group symmetry of the combined system has to be the same, we can use the analysis of the symmetry of the combined system which we performed before in order to obtain predictions on the symmetry of the growth shape of the islands. The point-group symmetry of the combined system tells us which directions are related by symmetry. As a consequence, the atomic structure of an (arbitrarily) complicated reconstructed step edge also has to be the same for directions related by symmetry. Thus also the growth speeds have to be the same for directions related by symmetry. As a result of this, finally the symmetry of the (steady-state) growth shape of the island has to obey the point-group symmetry of the combined system.

A 1D reconstruction present at a step edge can formally also break the symmetry. For instance, a mirror symmetry could be broken by a translational domain of a three times reconstruction of a reconstructed step edge. However, since in spite of this formally broken symmetry the whole step will advance with a specific speed, the orientation-dependent step speed is still maintained.

Another case where, in addition to the mathematical symmetry analysis the physical properties of the system come into play, are the probabilities for certain broken symmetries to occur. Previously we analyzed the number of cases (or probabilities) for which certain symmetries are maintained. As a consequence, one would assume to find these calculated a priori probabilities for the different symmetries also in the 
statistics of the measured symmetry of the islands. However, this is only true if islands with all possible translational domains occur with the same probability. Due to the physics in the system, such as lower total energy or higher barrier for the nucleation for a certain translational domain, the probabilities for the symmetries of the observed island shapes can be different than the a priori probabilities obtained from the symmetry analysis. On the other hand, such deviations from the a priori probabilities will give insight into the physics of the system (total energy or nucleation barriers).

\section{EXTENSIONS}

Domains of surface reconstruction can have, in addition to a translational shift, also a rotation relative to the substrate (rotational domain). A simple example would be domains of $(2 \times 1)$ and $(1 \times 2)$ on a simple cubic lattice. One can do all the steps of the symmetry analysis again instead of a pure translational shift for the case where one reconstruction lattice is rotated. The freedom of domain rotation can reduce further the symmetry of the combined system. For the examples given in here previously, we have considered only surface reconstructions with high symmetry, where rotational freedom does not affect the symmetry. However, in the case of less symmetric reconstructions [such as $(N \times M)$ ] one should include the rotational freedom in the symmetry analysis.

The analysis presented here can be extended to other cases. For instance, we considered only 2D islands of one atomic layer height. 2D islands of other heights can be easily included by just taking into account the correct shift of the island lattice relative to the substrate lattice for islands of multiple step heights. For example for three atomic layers high islands at a diamond (111) surface, the shift between the substrate layer and island layer induced by the substrate is zero. The periodicity length of the diamond structure in the (111) direction is three step heights.
While we considered only low-index surfaces, vicinal surfaces can be also analyzed. Due to the vicinality, the highest symmetry of a vicinal surface is $C_{s}$ with a mirror plane parallel to the direction of vicinality. This mirror plane can be broken, resulting in a $I$ symmetry, depending on the reconstruction.

Our analysis can be extended to cases in which the reconstruction on the island and the reconstruction on the substrate is different. For these cases the symmetry analysis has to be performed along the lines shown in the previous sections of this paper. We considered only pseudomorphic 2D islands. The analysis can be extended to epitaxial (not pseudomorphic) islands. In this case the intersection of the symmetry elements of the epitaxial island and the substrate has to be considered.

\section{CONCLUSIONS}

We analyzed the relationship between symmetry and shape (equilibrium shape or steady-state growth shape) of reconstructed $2 \mathrm{D}$ islands. We state that if some reasonable assumptions are fulfilled, the point-group symmetry of the (growth) shape of an island (crystal) is identical to the symmetry of the underlying crystal structure. Using this relation, a symmetry analysis can be used to predict the symmetry of the shape of the growing islands and equilibrium shapes. We present a method to analyze the symmetry of the combined system of a reconstructed island plus a reconstructed substrate from the symmetries of the subsystems. We introduce a method of symmetry analysis which is based on the determination of common symmetry elements of the two subsystems. We used this method to analyze as examples the symmetry of the shape of reconstructed 2D islands on reconstructed simple cubic (100), fcc (100), and diamond lattice (111) surfaces.

\section{ACKNOWLEDGMENT}

The authors acknowledge critical reading of the manuscript by H. P. Bonzel. *b.voigtlaender@fz-juelich.de

${ }^{1}$ B. K. Vainshtein and A. A. Chernov, Modern Crystallography (Nova Science Publishers, New York, 1988).

${ }^{2}$ J. C. Heyraud and J. J. Metois, Surf. Sci. 128, 334 (1983).

${ }^{3}$ A. Pavlovska, D. Dobrev, and E. Bauer, Surf. Sci. 326, 101 (1995).

${ }^{4}$ S. Surnev, K. Arenhold, P. Coenen, B. Voigtländer, H. P. Bonzel, and P. Wynblatt, J. Vac. Sci. Technol. A 16, 1059 (1998).

${ }_{5}^{5}$ S. Surnev, P. Coenen, B. Voigtländer H. P. Bonzel, and P. Wynblatt, Phys. Rev. B 56, 12131 (1997).

${ }^{6}$ K. Romanyuk, V. Cherepanov, and B. Voigtländer, Phys. Rev. Lett. 99, 126103 (2007).

${ }^{7}$ P. Curie, J. Phys. (Paris) 3, 401 (1894).

${ }^{8}$ F. E. Neumann, in Vorlesungen über die Theorie der Elastizität der festen Körper und des Lichtäthers, edited by O. E. Meyer (TeubnerVerlag, Leipzig, 1885).
${ }^{9}$ F. C. Frank and M. B. Ives, J. Appl. Phys. 31, 1996 (1960).

${ }^{10}$ A. Pimpinelli and J. Villain, Physics of Crystal Growth (Cambridge University Press, Cambridge, UK, 1998).

${ }^{11}$ G. Wulff, Z. Kristallogr. Mineral. 34, 449 (1901).

${ }^{12}$ C. Herring, Phys. Rev. 82, 87 (1951).

${ }^{13}$ International Tables for Crystallography, Volume A: Space Group Symmetry, edited by Theo Hahn (Springer, Berlin, 2005).

${ }^{14}$ L. Merz and K. H. Ernst, Surf. Sci. 604, 1049 (2010).

${ }^{15}$ V. G. Lifshits, K. Oura, A. A. Saranin, and A. V. Zotov, in Metals on Semiconductors, edited by A. P. Bonzel, Landolt-Börnstein, New Series, Group III, Vol. 42A1 (Springer, Berlin, 2001).

${ }^{16}$ K. Romanyuk, J. Myslivecek, V. Cherepanov, T. Sekiguchi, S. Yoshida, K. M. Itoh, and B. Voigtländer, Phys. Rev. B 75, 241309 (2007).

${ }^{17}$ V. Cherepanov, S. Filimonov, J. Myslivecek, and B. Voigtländer, Phys. Rev. B 70, 085401 (2004). 